\title{
Assessment of the applicability of the known principles of implementation of ground-based radio systems for problem solving of spacecraft control and identification
}

\author{
A. L. Polyakov, ${ }^{1, *}$, I. L. Afonin ${ }^{1}$, and D. A. Polyakov ${ }^{2}$ \\ ${ }^{1}$ Radio Electronics and Information Security Institute of Sevastopol State University \\ st. Universitetskaya, 33, Sevastopol, Russian Federation, 299053 \\ ${ }^{2}$ The military unit 17204, Gorodok St., Kolomna-1, Moscow Region, Russian Federation, 140401
}

\begin{abstract}
The creation of separate radio systems for solving the task of identifying spacecraft (SC) for uncontrolled radiation of on-board equipment requires considerable expenses, which makes it expedient to create hardware identification means as part of the already existing groundbased radio systems used for providing spacecraft control.
\end{abstract}

\section{Introduction}

Identification of active SC, i.e. SC with operating transmitting devices during the flight in the visibility range of radio engineering systems (RES) is not a difficult task [9]. However, for the majority of space systems for defense purposes, one of the characteristics of their operation is stealth, which, first of all, implies the shutdown of the SC on-board transmitters outside the visibility range of the RES. This circumstance greatly complicates the identification of these SCs.

Based on the performed analysis, it seems appropriate to develop an identification system that will improve the quality of functioning of the space control system.

The basis of such an identification system can serve as a ground-based RES with fullturn antenna devices [2]. This approach will allow creating a system with known parameters and providing all-weather fixation of SC from all flight directions.

At the same time, the identification and processing of spurious radiation signals of constantly functioning on-board equipment (OBE) units can be selected as identification parameters.

\section{The main part}

To solve this problem, it is necessary to estimate the possibility of receiving and processing parasitic radiation signals. From the equation of the radar is known [4]

$$
P_{c}=\frac{P_{n} G_{n}}{4 \pi H^{2}} A=\frac{Q_{n} A}{4 \pi H^{2}}
$$

*Corresponding author: al_polykov@inbox.ru 
where $\mathrm{P}_{\mathrm{c}}$ is the power of the received radio signal, $\mathrm{W}$;

$\mathrm{A}$ is the effective area of the ground-based system antenna;

$\mathrm{Pn}$ is the power of the emitted radio signal, W;

$\mathrm{Gn}$ is the onboard equipment gain coefficient;

$\mathrm{H}-\mathrm{SC}$ orbit altitude, $\mathrm{km}$;

$\mathrm{Qn}=\mathrm{Pn} \times \mathrm{Gn}-$ the SC coefficient.

Table 1. Effective antenna area of ground control complex.

\begin{tabular}{|c|c|c|}
\hline Antenna means & Wave ranges & Effective area $\mathrm{m}^{2}$ \\
\hline$D C A-1000$ & DM combined & 900 \\
\hline & DM & 650 \\
\hline & SM & 450 \\
\hline RT-32 & DM & 422 \\
\hline & SM & 407 \\
\hline RT-70 & $18 \mathrm{sm}$ & 2450 \\
\hline & $6 \mathrm{sm}$ & 2800 \\
\hline & $5 \mathrm{sm}$ & 2750 \\
\hline & $3,55 \mathrm{sm}$ & 2450 \\
\hline & $1,35 \mathrm{sm}$ & 1700 \\
\hline & $0,82 \mathrm{sm}$ & 850 \\
\hline
\end{tabular}

The main SC grouping is located at altitudes from 200 to 40,000 km [5]. The power value of the parasitic OBE radiation is about $1.2 \times 10-5 \mathrm{~W}$, and the gain coefficient of the onboard antenna is $1000[5,6]$. Considering the abovementioned, we obtain the height values of the identified SC orbit from expression (1) for ground-based systems with different effective antenna area (see Fig. 1).

The analysis of existing technical systems (Table 1) indicates the possibility of using antenna devices applied in these systems for SC identification tasks [6, 7].

In addition, this analysis allows us to conclude that the RES can be used with a receiving path sensitivity of $10^{-11}$ to identify $\mathrm{SC}$ in orbits about $150,000 \mathrm{~km}$.

Thus, the abovementioned studies determine the basis for creating an SC identification system.

The results of the conducted analysis show the possibility of SC identification. It is especially important to apply the obtained results in ground-based RES of single-point SC control and identification. Therefore, it is advisable to consider the features of existing outer space control systems and their applications for ballistic - navigation support of SC control.

The study of directions for improving the quality of functioning of the ground-based automated SC control complex (GACC) involves consideration of ballistic-navigation support tasks of SC control, as well as SC identification in visibility area of the REC used.

When solving problems associated with the provision of a SC flight program, it is necessary to measure constantly the parameters of their motion from the Earth. Traditionally, these tasks are solved by SC REC GACC trajectory means.

In this case, the determination of the current coordinate values and components of the velocity vector of the spacecraft with subsequent processing to obtain the necessary information about its movement is performed during the satellite flight in the visibility range of the RES. The parameters of the SC motion are determined during the control session due to the time required for the other modes of SC operation with the combined RES. 


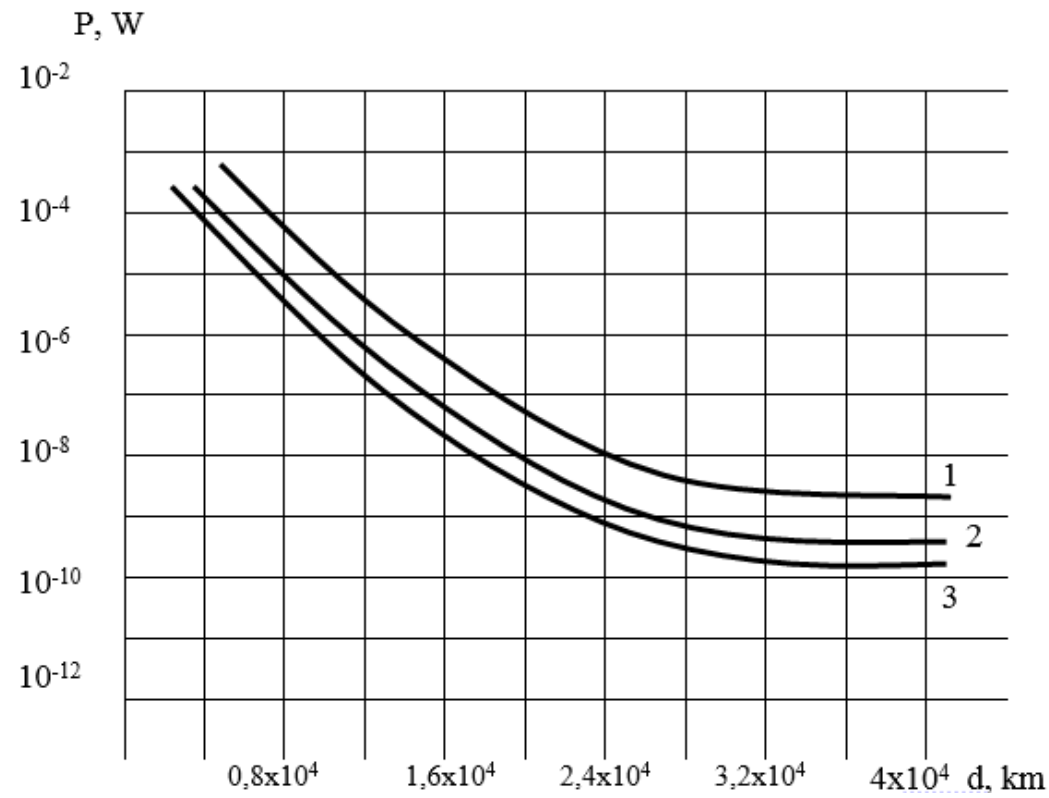

Fig. 1. Dependence of the height of the orbits of the identifying SC for ground-based antenna systems with different effective area, where 1 is an antenna complex with $\mathrm{Ae}=2500 \mathrm{~m}^{2} ; 2-$ antenna complex with $\mathrm{Ae}=1000 \mathrm{~m}^{2} ; 3-$ antenna complex with $\mathrm{Ae}=400 \mathrm{~m}^{2}$.

$$
t_{c y}=t_{T И}+t_{T M \Lambda}+t_{c u}+t_{\kappa p л},
$$

where $\mathrm{t}_{c y}$ is the time of the control session;

$t_{6}$ is the time interval of the SC flight in the visibility range of the REC, $t_{c y} \approx t_{6}$;

$t_{T И}$ - time interval of trajectory measurements. At the same time, it is known that $t_{c y} \approx t_{b} \approx t_{m u} ;$

$t_{\text {тми }}$ - time interval for receiving telemetric information on the status of BA SC;

$t_{c u}$ is the time interval for receiving special (scientific) information on the SC functional purpose;

$t_{\kappa р л}$ - time interval for issuing commands and temporary programs to the board and receiving from the board of receipts for passing these programs and commands.

It is known to be the trajectory means (TM) RES GBCC measuring the motion parameters of near-Earth SCs and interplanetary SCs, the systems include equipment installed onboard the $\mathrm{SC}$, and ground-based measuring equipment. It is also possible to create an identification system for detecting and tracking unknown and "silent" SC. Such a system has only ground-based measuring equipment and operates on the principle of conventional radar systems $[2,10]$. With the help of the trajectory means (TM) RES are determined: the distance to the SC (d), the angular coordinates (azimuth $(\alpha)$ and elevation $(\beta)$ ), the rate of change of range (radial velocity $(\dot{\alpha}$ and $\dot{\beta})$ ) and angular coordinates (angular velocity).

In this case, the TM range is determined depending on the SC type and may be in the range of hundreds of kilometers to hundreds of millions of kilometers. The required range of action is ensured by placing aboard spacecraft of transceivers with a radiation power of $0.25-50 \mathrm{~W}$ and a sensitivity of about $10^{-7}-10^{-10} \mathrm{~W}$ [5]; the use of terrestrial transmitters with a pulse power of up to $10 \mathrm{MW}$, and in the continuous mode of radiation - up to 100 $\mathrm{kW}$; increasing the sensitivity of ground receiving devices; the use of highly directional (AS) [4] VHF and EHF bands; increasing the noise immunity of the whole RES. 
One of the methods for reducing the error of RES measurements is to estimate the relative position of the trajectory means of the SC UCM. Traditionally, these tools are included in the combined RES command-path radio links that perform the SC control session during the $\mathrm{T}_{\mathrm{cy}}$ time. At the same time, for trajectory measurements of the RES in the composition of the low-level switchgear assembly of the SC are geographically separated by a much greater distance.

To measure the angular velocities, the existing trajectory means use at least three measuring points [8], forming at their placement two mutually perpendicular directions. Angular velocities in azimuth $\dot{\alpha}$ and elevation $\dot{\beta}$ are determined identically by the difference in Doppler frequencies [8]

$$
\begin{gathered}
\dot{\alpha}=\frac{c\left(\Delta \mathrm{f}_{Д}^{\prime}-\Delta \mathrm{f}_{Д}\right)}{\mathrm{f}_{0} \mathrm{~B} \sin \alpha}, \\
\dot{\beta}=\frac{\mathrm{c}\left(\Delta \mathrm{f}_{Д}^{\prime}-\Delta \mathrm{f}_{Д}\right)}{\mathrm{f}_{0} \mathrm{~B} \sin \beta},
\end{gathered}
$$

where $\left(\Delta f_{Д}^{\prime}-\Delta f_{Д}\right)$ the difference of Doppler frequencies, taken by two points;

$\mathrm{c}-$ the speed of light

To measure the range $d$ and angular coordinates $\alpha, \beta$, the SC positions in existing CSs, the phaseometric method is actively used [6]. In the RES trajectory means, the application of this method is based on measuring the phase shift of two interfering waves, which is proportional to the difference in the distances traveled, i.e.

$$
\Delta \varphi=\frac{2 \pi}{\lambda} \Delta \mathrm{d}
$$

where $\Delta \varphi$ - phase shift of interfering radio waves;

$\lambda$ - the length of the radio waves used;

$\Delta \mathrm{d}$ - the difference of the distances traveled by radio waves.

In the distance measuring RES systems, the phase difference is proportional to the distance to the $\mathrm{SC}$, and in the systems of measuring the angular coordinates it is the conventional SC position. The phase difference can be unambiguously measured only from 0 to $2 \pi$. The measurement of the phase difference in large limits leads to ambiguity in the measurements, which is eliminated by various methods $[5,7]$.

To determine the range to a SC via the Earth-to-SC radio link in known SC [6], a request signal is sent. This signal onboard the $\mathrm{SC}$ is relayed and radiated to Earth. Depending on the phase difference of the received signal relative to the request signal, the distance to the SC will be equal to the expression [7]

$$
d=\frac{\Delta \varphi \lambda}{4 \pi} .
$$

When measuring the angular coordinates, the onboard transmitter signal is received simultaneously by two or more ground-based points separated in space at a distance B. By determining the phase difference of the signals received by these points, you can calculate the direction to the SC using the formula [8]

$$
\beta=\arccos \frac{\Delta \varphi \lambda}{2 \pi \beta} .
$$

Currently, to solve the synchronization problem with extremely high accuracy, in addition to LBRS methods, three methods are being developed. The first uses the GLONASS and GPS navigation satellite systems, the second uses the synchronization signals broadcast via geostationary communication satellites (duplex method) and the third 
uses the exchange of laser signals through special reflectors installed on geostationary satellites (LASSO Lazer Synhronization from Stationary Orbit) [4].

The advantages of GPS-type systems are the low cost user equipment (with an extremely high cost of the space segment), global coverage and a virtually unlimited number of users. The disadvantages include the dependence of the comparison accuracy on the distance between the points and the fact that high accuracy can be obtained as a result of processing information from a large number of observations.

Potentially, one of the most accurate methods for comparing time scales is the LASSO method [4], but it requires the equipment of geostationary SC with special optical reflectors and very expensive ground-based equipment. In addition, the application of this method is limited by weather conditions, as a result of which it has not acquired wide practical application.

The duplex method of synchronization via satellite channel has high accuracy, as high accuracy is achieved during the measurement process and does not depend on the distance between the points being compared. This method is weatherproof. At the same time, the lack of a duplex method is the need to organize a satellite communications channel with high costs for acquiring satellite earth stations. However, this disadvantage is effectively overcome in connection with the improvement of space repeaters and the ability to use low-power earth stations $[5,6]$. For the case of the LBRS trajectory system, when the onboard RES of the reference $\mathrm{SC}$ in geostationary orbit and combining the functions of the space transponder is used as one of the measurement points, and a properly equipped ground-based substation is used as the second measurement point, the duplex method is particularly advantageous.

The advantages of the duplex method are confirmed by a comparative analysis of the existing synchronization methods performed in [7, 8]. The results of this analysis are summarized in table 2 .

Table 2. Results analysis of the existing synchronization methods.

\begin{tabular}{|l|c|c|}
\hline \multicolumn{1}{|c|}{ Method } & Accuracy, ns & Coverage \\
\hline GPS (common-view) & 10 & global \\
\hline GPS & 50 & global \\
\hline LASSO & 1 & Depending on satellite \\
\hline Duplex & 1 & global \\
\hline
\end{tabular}

When using single-point SC control technology, the accuracy of the RES trajectory measurements and the speed of processing information about the SC space-time position are significantly reduced. To solve this problem, it seems reasonable to use spatially separated means with one ground-based RES. Radio astronomy systems can be used as such trajectory systems. In particular, radiointerferometric measurements of space objects using signals of uncontrolled radiation of onboard equipment that carry trajectory and identification information about $\mathrm{SC}$ can be a promising direction.

Thus, the assessment of the applicability of the known methods of functioning of the existing SC UCM indicates the need to develop a methodology for creating promising CSs in the direction of researching nonlinear processes in the RES that use the radiointerferometric methods of the space control system BNP and identifying SC that are in view area of these RETs for one-item construction of TCUs. and processing of uncontrolled radiation onboard equipment.

\section{Conclusion}

The SC identification as an integral part of a system for monitoring and analyzing a space situation can be carried out by a radio engineering complex having a full-turn antenna with a relatively large effective range and a high sensitivity of the receiving path of the 
order of $10^{-7}$ Watts. In this case, the signals used to identify the SC may be signals of uncontrolled radiation from the master oscillator of the onboard REC of this device, which, due to the "leakage" effect through antenna switches, allow ground-based means to "listen to silent" SC that are in visibility range of these means.

To improve the efficiency and quality of the SC identification, and the entire system of control and analysis of the space situation, the relevant task is to develop an appropriate method for the SC identification and the algorithms implementing it.

Thus, the development of theoretical foundations and practical recommendations for creating a ground-based RES that receives and processes signals from UCM IG BRES to identify SC in the visibility range of this system, as well as study its characteristics and applicability in promising rocket-space complexes to ensure the required values The quality indicators of the systems for monitoring and analyzing the space situation are timely, relevant and of great defense importance. Therefore, the solution of the scientific problem and the research questions that comprise it provides the possibility of achieving the goal of given study.

\section{References}

1. S.V. Kozelkov, Development of scientific and technical proposals to improve the noise immunity of antenna-receiving devices of rocket and space complexes: Dis. cand. tech. sciences: 20.02.14. Kharkov (1991)

2. S.V. Kozelkov, V.G. Stadchenko, N.V. Nyukin, and S.V. Shurygin, "On the issue of creating a spacecraft identification complex", in: Information Systems. Iss. 3 (11). Kharkiv: NASU, PANI, KHVU, pp.103-106 (1998)

3. R.V. Bakitko, M. B. Vasiliev, A. S. Vinitsky et al. Radio systems of interplanetary spacecraft. Ed. by A. S. Vinitsky. Moscow, Radio i svyaz (1993)

4. L.I. Gusev and E.P. Molotov, Terrestrial space tracking network for deep-space spacecraft and high-altitude weather satellite satellites. Space Bulletin, 2, No. 1 (1995)

5. S.V. Kozelkov, "Application of a planetary radar for spacecraft navigation problems", Systems of information processing. Is. 1 (5). Kharkiv, NASU, PAN, KHVU, pp.139142 (1999)

6. I.I. Livshits, V.M. Rozhkov, and B.A. Ryabov, "Using a satellite for communication in the range of millimeter waves", Foreign Radio electronics. No. 5, pp. 41-49 (1987)

7. M.A. Ivanov and S.V. Kozelkov, Analysis of the conditions of use of antenna devices of microwave and high frequency range on satellite transponders. Moscow, In CIVTI MO USSR, is. 10, No 4238, B1384. (1989)

8. S.V. Kozelkov, "Guidance and auto-tracking systems for antenna devices of microwave and EHF radio links", Scientific and practical aspects of managing space and earth groups, features of their application. USSR Ministry of Defense (1989)

9. Handbook of antenna technology. Ed. J. Feld and E. G. Zelkin. Moscow, IPPRZhR, 1 (1997)

10. A.I. Pogorelov, “Analysis of Systems for Processing Complex Spatial - Time Signals", Signal Processing in Radio Systems. Kharkov, KhAI, pp. 164-173 (1988) 